\title{
Autism Spectrum Disorders, is it Under Reported In Third World Countries
}

\author{
Amar Al-Shibli ${ }^{1}$ and Osama Hamdoun*2 \\ ${ }^{1}$ Consultant Pediatrician, Tawam Hospital, UAE \\ ${ }^{2}$ Pediatric resident, Tawam Hospital, UAE \\ *Corresponding author: Osama Hamdoun, Pediatric resident, Tawam Hospital, UAE \\ To Cite This Article: Osama Hamdoun. Autism Spectrum Disorders, is it Under Reported In Third World Countries. Am J Biomed Sci \& Res. 2019 \\ - 4(4). AJBSR.MS.ID.000818. DOI: 10.34297/AJBSR.2019.04.000818
}

Received: July 30, 2019 | Published: August 12, 2019

\section{Editorial}

Autism spectrum disorders (ASD) is defined as a pervasive developmental disorder characterized by impairment of social communication, repetitive and restrictive patterns of behavior, interests, and activities.

The incidence of autism steadily increasing in the past decades in both developing and developed countries. Worldwide prevalence of ASD is 7.6 per 1000 ( 1 in 132). The prevalence of ASD in Europe, Asia, and the United States ranges is 1 in 40 to 1 in 500 while comparing developed countries to developing countries its notable that the incidence is lower in developing countries, an example of developing country is China which has the incidence of 1.1 in every 1,000 children. However, this is not reflecting the reality, in fact the lower incidence is due to under reporting of the affected children.

It is well known that autism is a multi-factorial disease, which is contributed to genetic or/and environmental causes, unfortunately so far, no fingers cannot be pointed toward a clear reason. A community-based case control study was conducted in the US on 2961 individuals with a diagnosis of autism spectrum disorder, the study gave additional evidence that pesticides are related to autism and intellectual disability. Interestingly, from the pesticides which were studied, there is 2 commonly used medications in the treatment of head lice and scabies, which are malathion and permethrin. Another unique study done last year, for first time it is shown high levels of aluminium in brain tissue of 5 children with autism, this study although it is small sample, but it attributed the incidence of autism to the high levels of aluminium deposition in brain tissue and this needs to be further studied. there were other reports trying to link ASD with other environmental factors, drugs, diet and vaccinations; However, non-has strong evidence. There are probably other factors in other areas of the world that were not well studied.

ASDs may significantly limit the capacity of an individual to conduct daily activities and participate in society. ASD often neg atively influence the person's educational and social attainments as well as employment opportunities. While some individuals with ASD are able to live independently, others have severe disabilities and require life-long care and support.

The diagnosis of $\mathrm{AD}$ is usually clinical and there are criteria like DSM V criteria that includes: persistent difficulties in the social use of verbal and nonverbal communication, functional limitations social participation, social relationships, academic achievement, or occupational performance, individually or in combination. the onset of the symptoms is in the early developmental period, symptoms are not attributable to another medical or neurological condition.

Some people with ASD can live independently, others have severe disabilities and require life-long care and support and for those the earlies psychosocial interventions the better the outcome.

Despite of the bad consequences of late detection of autism, still many cases have delayed diagnosis as a result there is delay in treatment, which will have huge impact on the prognosis. Delayed diagnosis may be related to lack of awareness, fear from social stigmata and poor access to medical care, especially in developing countries. Regardless the reason, this will cause a huge financial and social burden on the family and government.

ASD has high association with lower IQ and mental, which will need extra attention management and early intervention which is found to be very effective specially when initiated below the age of 2 years includes both pharmacologic and non-pharmacologic treatments. the non-pharmacologic therapy includes group therapies, which encourage autistic patients and stimulates them to express their feelings, additionally, Improve the social skills and interactions with other people. Other modes of therapy are psychotherapy, occupational and speech therapy. Psychotherapy is crucial due to the high incidence of comorbid mental illness such as Attention-Deficit/Hyperactivity Disorder (ADHD) and depression in 
these patients, unfortunately these children are more risk of abuse and neglect. Occupational therapy is needed to teach the child his daily activities, such as dressing, writing and eating. speech and language therapy is from the major pillars of treatment, which is needed to teach communication with others
In conclusion, ASD is a prevalent disorder that will have bad outcomes if diagnosed late. The incidence and prevalence in the developing counties is not clear and this might affect the diagnosis, management and ultimately the outcome and the impact of the disorder. More studies for the incidence, prevalence, possible and management. 